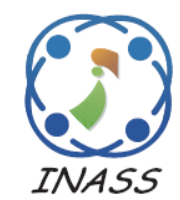

\title{
Adaptive Weighted Improved Discrete Particle Swarm Optimization for Minimizing Load Balancing Index in Radial Distribution Network
}

\author{
Manikandan Subramaniyan $^{1^{*}}$ Sasitharan Subramaniyan ${ }^{2} \quad$ Moorthy Veerasamy $^{3}$ \\ Viswanatha Rao Jawalkar ${ }^{4}$ \\ ${ }^{1}$ Sathyabama University, Chennai, India \\ ${ }^{2}$ ABB Global Indus. \& Ser. Ltd, Chennai, India \\ ${ }^{3}$ Swarnandhra College of Engineering \& Technology, Narsapur, India \\ ${ }^{4}$ VNR Vignana Joythi Institute of Engineering \& Technology, Hyderabad, India. \\ * Corresponding author's Email: svmmani79@gmail.com
}

\begin{abstract}
In this paper a metaheuristic based newfangled adaptive weighted improved discrete particle swarm optimization (AWIDPSO) algorithm is applied to minimize the load balancing index in radial distribution network reconfiguration (RDNR) problem. It is devised as extremely nonlinear and multimodal optimization problem under practical constraints. In order to improve the solution quality the constraint violations are augmented with objective function. Further, adaptively varying inertia weight increases the possible solution in the global search space and the proposed algorithm has obtained the optimal solution within lesser executing time. In this study, 33-bus system is analyzed for optimal network reconfiguration using the developed framework. Comparison of the simulated results with the results of well known prudent optimization technique confirms the applicability of the AWIDPSO algorithm for RDNR problem.
\end{abstract}

Keywords: Metaheuristic algorithm, Radial distribution system, Network reconfiguration, Load balancing index, Voltage profile improvement.

\section{Introduction}

In the rapidly growing research field emerging optimization technique facilitates to find a feasible solution for complex engineering problems. In which metaheuristic optimization is one that deals with optimization problems using metaheuristic algorithms. These are the simplest sense, gradientfree, non-deterministic, not problem specific and have been inspired by the natural selection process. Moreover, randomness features, intensification, and diversification driving forces of the metaheuristic algorithms bring the control parameters of the nonlinear problem to the edge, whereas, mathematical methods difficult to produce an accurate result. So, the metaheuristic optimization is to be an effective tool to solve nonlinear problems
[1]. As stated above it is not a problem specific, has less hold on initial solution point and tendency to solve large-scale and any kind of complex engineering problem, the researchers are motivated to solve distribution network reconfiguration (DNR) problem. It can be mathematically formulated as an optimization problem subjected to various operational constraints to ascertain radiality in the distribution network optimally that reduces the power loss and minimizes load balancing index [2$3]$ or maximize the benefits under the normal operation conditions [4].

In this context some metaheuristic algorithms such as genetic algorithm (GA) [5], a new hybrid evolutionary algorithm (EA) based on the combination of the honey bee mating optimization (HBMO) and the particle swarm optimization (PSO) [6], plant growth simulation algorithm [7], modified 
shuffled frog leaping algorithm (MSFLA) [8], fuzzy based multiobjective ant colony search-based algorithm (FMACSA) [9], PSO with a big bang-big crunch algorithm BB-BCA [10] and invasive weed optimization (IWO) [11] have been enhancing the global search capability in the search region for minimizing the load balancing index of distribution network.

Alternatively, Myint has applied exhaustive search techniques for minimizing power loss and improving voltage profile of the distribution networks are located in Dagon Seikkan Township in Yangon [12]. Afterwards the PSO- BB-BCA algorithm was applied DNR problem in the presence of capacitor placement [13] whereas; artificial bee colony (ABC) algorithm has been employed to minimize distribution power loss minimization in the existence of distributed generation and capacitor [14]. Rameshkumar et al., has applied discrete particle swarm optimization (DPSO) algorithm for solving permutation flow shop scheduling problems with the objective of minimizing the make-span [15]. Followed, Mingyu Li et al., has proposed an improved discrete particle swarm optimization (IDPSO) algorithm for high-speed train assembly sequence planning where the particles have a larger probability learning coefficients that improves the convergence rate [16].

As far as the state-of-the art, literature, it is understood that a proper balance between intensification and diversification of a metaheuristic technique facilitates to find a feasible solution. In [6] global search behavior of PSO is combined with local search behavior of HBMO whereas, in $[10,13]$ the exploration ability of BB-BC is integrated with the exploitation ability of PSO. Despite, these algorithms have found optimum radial structure; it is not an end global solution to DNR problem.

The DNR is a complex combinatorial problem which involves discrete control variables (position of the tie and sectionalizing switches) that should be evaluated each and every iteration to find the optimal radial structure. As, [15] and [16] proven its ability for finding good quality of solution it is motivated to optimize the above stated control variables, but the larger learning coefficients might tune the exploration ability of the algorithm that has improved convergence rate only.

Therefore, in this paper an adaptive weighted improved discrete particle swarm optimization (AWIDPSO) is proposed for finding feasible solutions to DNR problem. In which the dynamically varying weight coefficient of the evolution and aggregation, speed factor provides necessary strategic balance between intensification and diversification to find out the best solution quickly and ride over the local optima. The highlights of this work are:

- The IEEE 33 bus system data is suitably incorporated in the developed AWIDPSO algorithm.

- The constraint violation is properly handled using appropriate mechanism.

- A newfangled DNR framework is proposed to minimize load balance index.

- The optimal solution is obtained very shorter process time.

- The versatile characteristics of AWIDPSO algorithm are explored by comparing its solution with prudent optimization.

The paper is organized into six sections, in the next section, mathematical formulation of AWIDPSO algorithm as an optimization tool is briefed. Section 3 describes the DNR problem, whereas; section 4 deals application of AWIDPSO algorithms for finding the optimal radial operating structure. The numerical simulation results are presented and have compared in section 5. Finally, the conclusion is presented in the last section.

\section{AWIDPSO algorithm}

The traditional particle swarm optimization (PSO) is proposed by Eberhart and Kennedy inspired by the social behavior of organisms such as bird flocking and fish schooling. In basic PSO the particles are initialized randomly, then the velocity of the agents is updated using an integrated exploration and exploitation principle, with this information the position of the agent is updated towards the best solution in the feasible region during iterative process. Though, PSO algorithm has converged quickly in the beginning stage of the optimization and often falls into local optimum at the end while solving complex engineering problems.

Followed, improved discrete particle swarm optimization (IDPSO) algorithm has been proposed by changing the parameters such as the inertia weight, the learning factors and the size of the population. In which the value of the inertia weight in the velocity update equation decreases linearly from an initial value $\left(w_{\max }\right)$ to a final value $\left(w_{\min }\right)$. It improves global search capability of the IDPSO and make IDPSO algorithm find the fittest area quickly. However, the optimization accuracy of the above algorithms would decrease for the nonlinear optimization problem.

The inertia weight $w$ plays an important role in updating particle's velocity that improves the 
convergence rate and controls the exploration and exploitation process. The selection of bigger valued inertia weight accelerates the particles in the global search space quickly and a smaller valued inertia weight accelerates the particles in the local search space steadily. Therefore, a dynamically changing inertia weight strategy is introduced in the IDPSO algorithm wherein the inertia weight changes from a bigger value to a smaller value adaptively and achieves a comparatively optimal accuracy and speedy convergence; hence it is named as adaptive weighted IDPSO (AWIDPSO) algorithm. Then, the velocity and position of the particle in the AWIDPSO algorithm are renewed after the iteration as follows.

$$
\begin{aligned}
v_{i}(t+1)= & {\left[w_{\max }-e \cdot w_{e}+s \cdot w_{s}\right] v_{i}(t) } \\
& +c_{1} \text { rand }\left(p_{\text {best }, i}-x_{i}(t)\right) \\
& +c_{2} \text { rand }\left(g_{\text {best }, i}-x_{i}(t)\right) \\
x_{i}(t+1)= & x_{i}(t)+v_{i}(t+1)
\end{aligned}
$$

Where,

Evolution speed factor $(e)$

$$
=\frac{\min \left(g_{\text {best }}(t), g_{\text {best }}(t-1)\right)}{\max \left(g_{\text {best }}(t), g_{\text {best }}(t-1)\right)}
$$

Aggregation degree factor $(s)$

$$
=\frac{\min \left(g_{\text {best }}(t), \bar{p}_{\text {best }}(t)\right)}{\max \left(g_{\text {best }}(t), \bar{p}_{\text {best }}(t)\right)}
$$

Current mean of all individual optimal value

$$
\bar{p}_{\text {best }}(t)=\frac{1}{N} \sum_{t=1}^{N} p_{\text {best }, i}(t)
$$

and

$w_{\max }$ is maximum inertia weight

$w_{e}$ is weight coefficient of " $e$ "

$w_{s}$ is weight coefficient of " $s$ "

$c_{1}$ and $c_{2}$ are nonnegative learning factors

It can be seen from the velocity update formula (1) that the value of current velocity inertia weight coefficient adaptively changes according to the realtime state of all particles. As the iteration time increases, $c_{l}$ is reduced and $c_{2}$ is increased gradually for obtaining strategic balancing between global and local search. At the premise of diversity assurance, AWDPSO algorithm can effectively improve the algorithm convergence speed and achieve a relatively optimal accuracy and speed.

\subsection{Pseudo code of AWIDPSO}

Step-1: Initialize the parameters: Swarm size, max no. of cycles, each agent's position and velocity, local-best position and global best position, nonnegative learning factors, maximum inertia weight of the evolution speed and aggregation factors.

Step-2: Updating and Fitness evaluation.

Step-3: FOR iteration number $t=1$; REPEAT

Step-4: Renew the velocity and position of each agent according to Eq. (1) and (2) respectively, and verify the new position $x_{i}(t+1)$ is in a feasible range

$$
\begin{aligned}
& \text { If } x_{i}(t+1) \text { is better than } x_{i}(t) \\
& p_{\text {best }, i}=x_{i}(t+1) \\
& \text { If } x_{i}(t+1) \text { is better than } g_{\text {best }, i} \\
& g_{\text {best }, i}=x_{i}(t+1)
\end{aligned}
$$

Step-5: $t=t+1$

Step-6: UNTIL iteration = Maximum;

Step-7: STOP

Step-8: OUTPUT $=g_{\text {best }, i}$

\section{Problem formulation}

The electrical distribution system is a widespread network and group of networks are interconnected as meshed structure. These are configured as a radial in structure using sectionalizing switch/ tie switch under normal operating condition to transfer the load among the feeders and to facilitate simple protective scheme. Therefore, it is stated that the DNR is a task of changing the structure of the distribution network to maintain the optimum radial operating structure. In this paper the DNR problem is formulated as a complicated combinatorial, non-differentiable constrained optimization problem to minimize the load balancing index.

\subsection{Objective function}

Load imbalance is often happening in a distribution system due to a mixture of different types of loads and its pattern. For the purpose of load balancing, the radial structure of the distribution feeders can be modified from time to time in order to reschedule the load currents more efficiently for loss minimization. The load-balancing problem comprises two indices, one is line usage index (LUI) and another is a system load balancing index (LBI). The first one is defined as a measure of what portion of the branches has been loaded without exceeding the rated capacity of that branch. The LUI of $i^{\text {th }}$ branch is calculated using Eq. (6). 


$$
\text { Line usage index }=\frac{I_{i}}{I_{i}^{\max }}
$$

Where, $I_{i}$ and $I_{i}^{\max }$ are $i^{\text {th }}$ branch current and its maximum current carrying capacity in ampere. Later is used to measure the overall system loading and it has minimized by optimizing the LUI. In other words, all the line usage indices are set to be more or less the same value and are also nearly equal to the system load balancing index and it can be written by Eq. (7).

$$
Y=\left[\frac{I_{1}}{I_{1}^{\max }} \frac{I_{2}}{I_{2}^{\max }} \cdots \frac{I_{i}}{I_{i}^{\max }} \cdots \frac{I_{n}}{I_{n}^{\max }}\right]
$$

Now the system LBI is defined as:

$$
L B I=\operatorname{Var}(Y)
$$

Where, Var represents the variance operation. However, the smaller value of the LUI index indicates that the load balancing has been conducted more efficiently. Therefore, the objective of minimizing load balancing index (LBI) is expressed as follows:

$$
\text { Minimize (LBI) }
$$

\subsection{Constraints}

Bus voltages after reconfiguration should be in their permissible range specified by the system operator:

$$
V_{\min } \leq V_{j} \leq V_{\max } ; j \in N_{\text {bus }}
$$

Where, $V_{\min }$ and $V_{\max }$ are minimum and maximum node voltage of $j^{\text {th }}$ node in pu.

The current in each branch must satisfy the branch's capacity.

$$
\left|I_{i}\right| \leq I_{i}^{\max } ; i \in N_{b r}
$$

\section{Computational flow}

The conquest step by step procedure of implementing AWIDPSO for DNR problem is described as follows:

\section{Step 1: Initialization of particles}

The tie-switches to be opened for maintaining feasible radial topology are control variables to the AWIDPSO. These control variables are integer numbers, the number of control variable is the sum of the number of tie switches which is randomly chosen from each fundamental loop. Thus, the number of fundamental loops $\left(N_{L}\right)$ is determined using Eq. (12).

$$
N_{L}=N_{b r}-\left(N_{b u s}-N_{s s}\right)
$$

\section{Step 2: Structure of initial population}

As the control variable is an integer number and have been selected one from each loop it can be stated that the number of fundamental loops is equal to the number of tie-switches to be opened for maintaining radial structure. Then uniformly distributed random integer is generated using Eq. (13).

$$
\begin{array}{r}
X_{i, j}=\text { rand } \operatorname{int}\left(1,1,\left[1, T S_{j}\right]\right) ; \\
i \in N P \text { and } j \in N_{\text {tie }}
\end{array}
$$

Then the randomly generated $N P$ populations have initially structured as matrix and it is represented in Eq. (14).

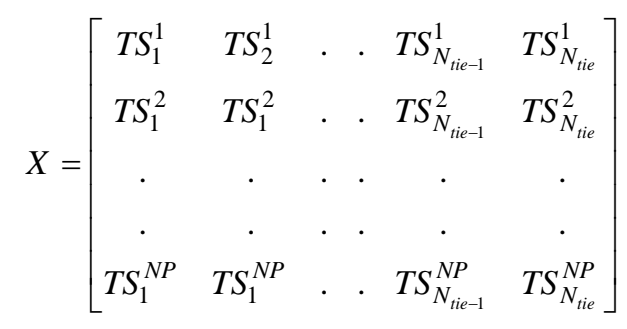

\section{Step 3: Estimation of augmented objective function}

From the initial position of the population, the radial structure has been realized through a suitable mechanism, and then a standard distribution load flow algorithm is executed and the value of fitness function is calculated from its solution. In order to handle constraint violation an augmented objective function (AOF) is derived using (15), which is the aggregate of the fitness function and the squared sum of the violated voltage $\left(S_{C V}\right)$ and current constraints $\left(S_{C I}\right)$ with a high valued scalar multiplier. Further, this mechanism converts the primal constrained problem into an unconstrained problem and guides the search process towards the desirable solution.

$$
A O F=\left(\text { Objective }+\lambda_{V} S_{C V}+\lambda_{I} S_{C I}\right)
$$

The $S_{C V}, S_{C I}$ values can be determined as follows: 


$$
\begin{array}{r}
S_{C V}=\sum_{j=1}^{N_{\text {bus }}}\left(\max \left\{0, V_{\min }-V_{j}\right\}^{2}\right. \\
\left.+\max \left\{0, V_{j}-V_{\max }\right\}^{2}\right) \\
S_{C I}=\sum_{l=1}^{N_{b r}}\left(\max \left\{0, I_{l}-I_{\max }\right\}^{2}\right)
\end{array}
$$

\section{Step 4: Evaluation of Fitness and of best position}

The fitness value of all individuals of the current particle set matrix $(X)$ is calculated using (18). The fitness of $i^{\text {th }}$ individual is chosen as the best local position $\left(p_{\text {best }}\right)$ and the individual that has the maximum objective value should be chosen as its neighborhood i.e. global position $\left(g_{\text {best }}\right)$.

$$
\text { fit }_{i}=A O F
$$

Step 5: Updating particle position for optimal solution

The velocity of the $i^{\text {th }}$ individual should be updated in accordance with (1) and the modified position for the $i^{\text {th }}$ individual should be calculated based on (2). The new position of each particle may violate allowable ranges and it is limited to the respective range.

\section{Step 6: Fitness re-estimation}

With the new position of each control variable, a standard load flow is run and the AOF is calculated and followed the best position of fitness is evaluated to identify a global best solution.

\section{Step 7: Stopping criterion}

If iter <max cycle; AOF is estimated. Otherwise, the AWIDPSO algorithm terminates

\section{Result and discussion}

Standard IEEE 33-bus, $12.66 \mathrm{kV}$ and $100 \mathrm{MVA}$ base radial distribution system is considered for this study. It consists of 32 sectionalizing switches and 5 tie-switches namely $33,34,35,36$ and 37 . The total load conditions are $3715 \mathrm{~kW}$ and $2300 \mathrm{kVAr}$, the initial power losses and minimum per unit voltage are $202.67 \mathrm{~kW}$ and 0.913 , respectively. The complete particulars can be found in [2]. The AWIDPSO algorithm is developed in the MATLAB 7.9 platform and is implemented on an Intel ${ }^{\circledR}$ CoreTM i5-4210C CPU, 1.70GHz, 4-GB RAM digital computer. The DNR problem is simulated using AWIDPSO for minimizing load balancing index. The simulated result of the test system is compared with the previous methods in order to substantiate the robustness of the algorithm.

\subsection{Optimal reconfiguration}

To prove the potentiality of the proposed AWIDPSO for minimizing load balancing index, it is executed with suitable control parameters and the tie-switch position is optimized over 100 independent iterations. As a result, the independent optimal tie-switch position, load balance index, node's minimum voltage and computational times are presented in Table 1.

It is recognized that there are three new [7, 11 and 31] branches are opened optimally at the same time tie-switches 33, 35 and 36 have been closed simultaneously to maintain radial structure. The minimum node voltage is found $0.9428 \mathrm{p} \mathrm{u}$., whereas LBI was 0.33820 and the algorithm has optimized the problem in 11.18 seconds. The corresponding network reconfiguration is illustrated in Fig. 1.

\subsection{Solution quality improvement}

Table 2 shows the comparison of optimal tieswitches position, LBI and its improvement that has obtained by FMACSA [9], IWO [11], HBB-BC [10] and proposed AWIDPSO. The proposed method was minimized the LBI more extent and it has improved $78.54 \%$ from initial state while $55.13 \%$, $9.36 \%$ and $36.67 \%$ with respect to FMACSA [9], IWO [11] and HBB-BC [10] respectively. Further, it is noticed that the voltage regulation was $6.17 \%$, which is lesser than other methods. Moreover, the algorithm exhibits stable and steady characteristic once again and is given in Fig. 2.

In fact, this algorithm indulges dynamically varying evolution speed and aggregation degree, factors to increase the global search ability to reduce the problem of the algorithm being trapped into the local optimum problem. However, the updating of the $w$ using adaptively changing strategy is not only related to the number of iterations, but also closely related to the algorithm dynamic performance of all particles. Further, it could enhance the population diversity and hence the proposed algorithm provides better solution than other methods.

Table 1 Possible optimal tie-switch position

\begin{tabular}{|c|l|l|l|}
\hline $\begin{array}{c}\text { Optimal Tie- } \\
\text { switches }\end{array}$ & LB index & $\mathrm{V}_{\text {Min }}(\mathrm{pu})$ & $\begin{array}{c}\text { Comp. } \\
\text { time (s) }\end{array}$ \\
\hline $7,11.31,34,37$ & 0.033820 & 0.9428 & 11.18 \\
\hline $6,31,34,35,37$ & 0.034130 & 0.9420 & 11.30 \\
\hline $6,11,31,34,37$ & 0.036320 & 0.9417 & 11.40 \\
\hline $6,9,31,34,37$ & 0.036810 & 0.9413 & 11.38 \\
\hline $6,9,14,31,37$ & 0.036800 & 0.9415 & 11.28 \\
\hline
\end{tabular}




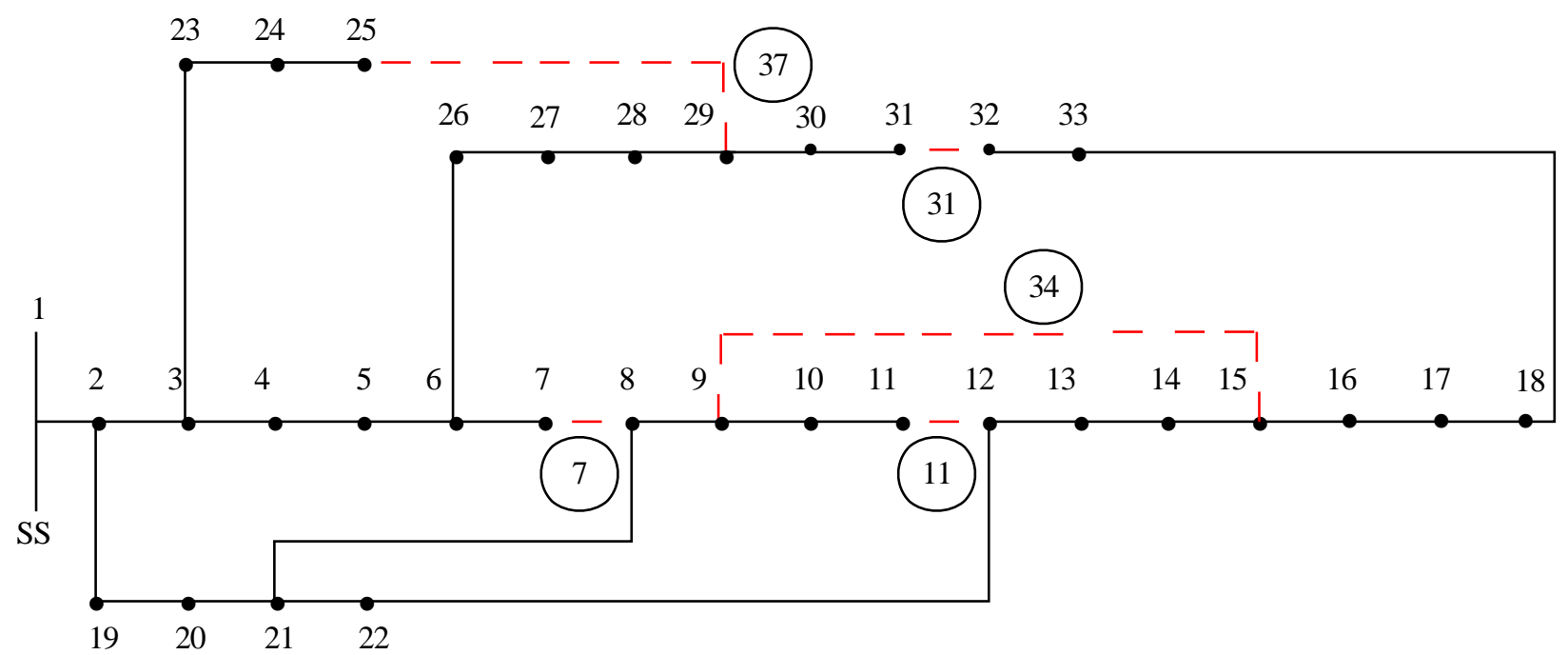

Figure.1 Optimal reconfiguration for 33 bus system

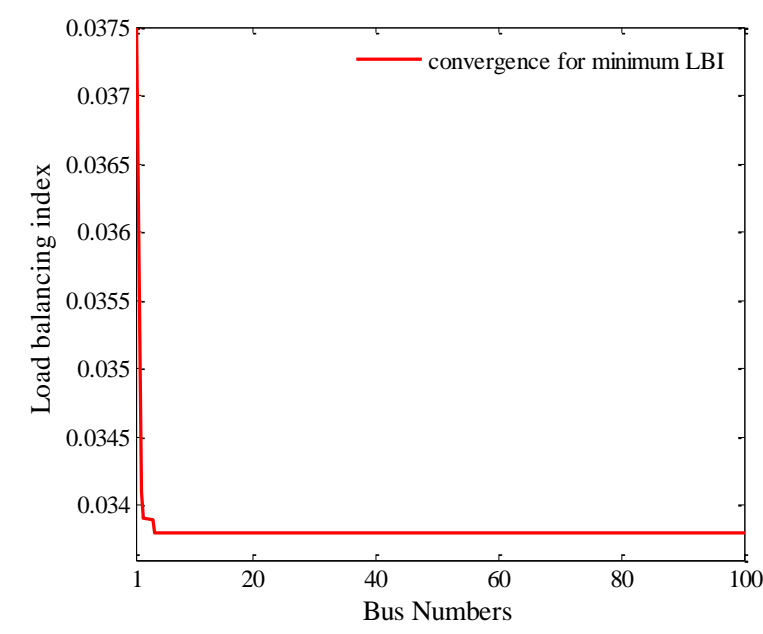

Figure.2 Convergence characteristics of AWIDPSO

The branch current profile corresponding to the minimum LBI is presented in Fig. 3. Where, the branch current before and after network reconfiguration is compared and it is revealed that the branch current satisfies the line ampacity after reconfiguration by the proposed algorithm for the entire node. Further, power flows and power loss at branch level have been obtained by the proposed algorithm is compared with the values that were obtained at initial state in Fig. 4 and Fig. 5 respectively. The power flow in each branch is reduced after reconfiguration that shows the feeders are free from overloading and moreover, facilitates to carry additional load in future. The power loss in each branch after reconfiguration is reduced almost, except at 3, 4, 5, 6, 18, 20, 21, 25, 26, 27, 28, 30, 32, 33,35 and 36, where the losses are increased because of shifting of loads onto these feeders.

\subsection{Statistical analysis}

The feasible solutions of the DNR for minimum LBI over 30 independent run are statistically analyzed and the test results are presented in Table 3, it contains the best, average and worst values among the final solutions that have obtained by AWIDPSO and HBB-BC [10] algorithms. As the AWIDPSO algorithm has the lowest standard deviation, the best solution is clustered closely around the average value and it shows the ability of the proposed algorithm in finding optimum solutions.

Table 2. Feasible solution for minimum load balancing index

\begin{tabular}{|l|c|c|c|c|c|}
\hline \multicolumn{1}{|c|}{ Methods } & $\begin{array}{c}\text { Optimal } \\
\text { Tie-switches }\end{array}$ & LB index & $\begin{array}{c}\text { \% of } \\
\text { improvement }\end{array}$ & $\begin{array}{c}\text { Switches } \\
\text { changed }\end{array}$ & $\begin{array}{c}\text { \% of Voltage } \\
\text { regulation }\end{array}$ \\
\hline Initial State & $33,34,35,36,37$ & 0.157567 & --- & --- & \\
\hline FMACSA[9] & $37,31,14,9,6$ & 0.075370 & 52.17 & 4 & 7.65 \\
\hline IWO[11] & $7,9,14,31,37$ & 0.037314 & 76.32 & 4 & 8.23 \\
\hline HBB-BC[10] & $11,28,31,33,34$ & 0.053407 & 61.11 & 3 & 8.31 \\
\hline AWIDPSO & $7,11,31,34,37$ & 0.033820 & 78.54 & 3 & 6.07 \\
\hline
\end{tabular}




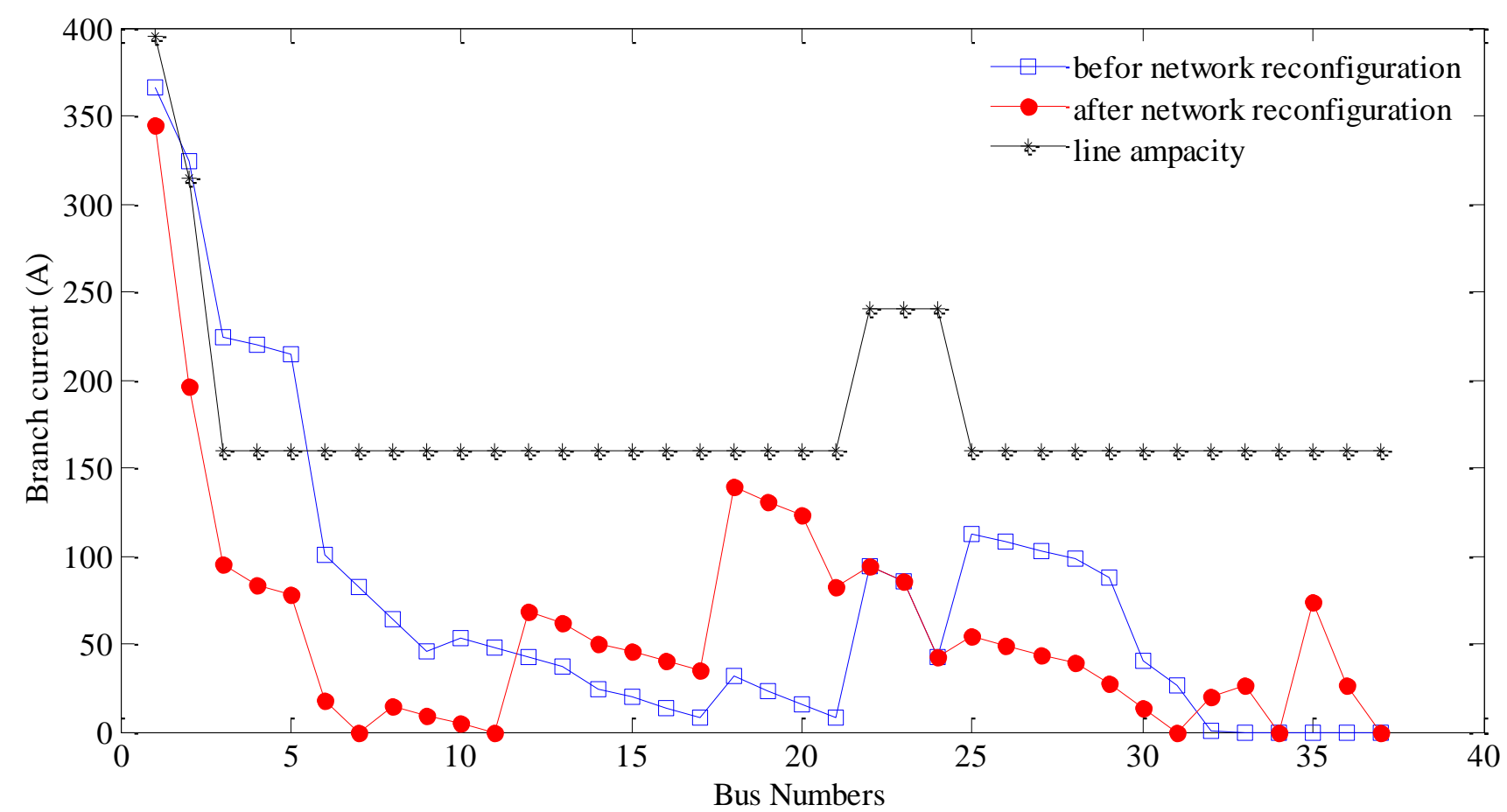

Figure.3 Branch current profile for 33 bus system

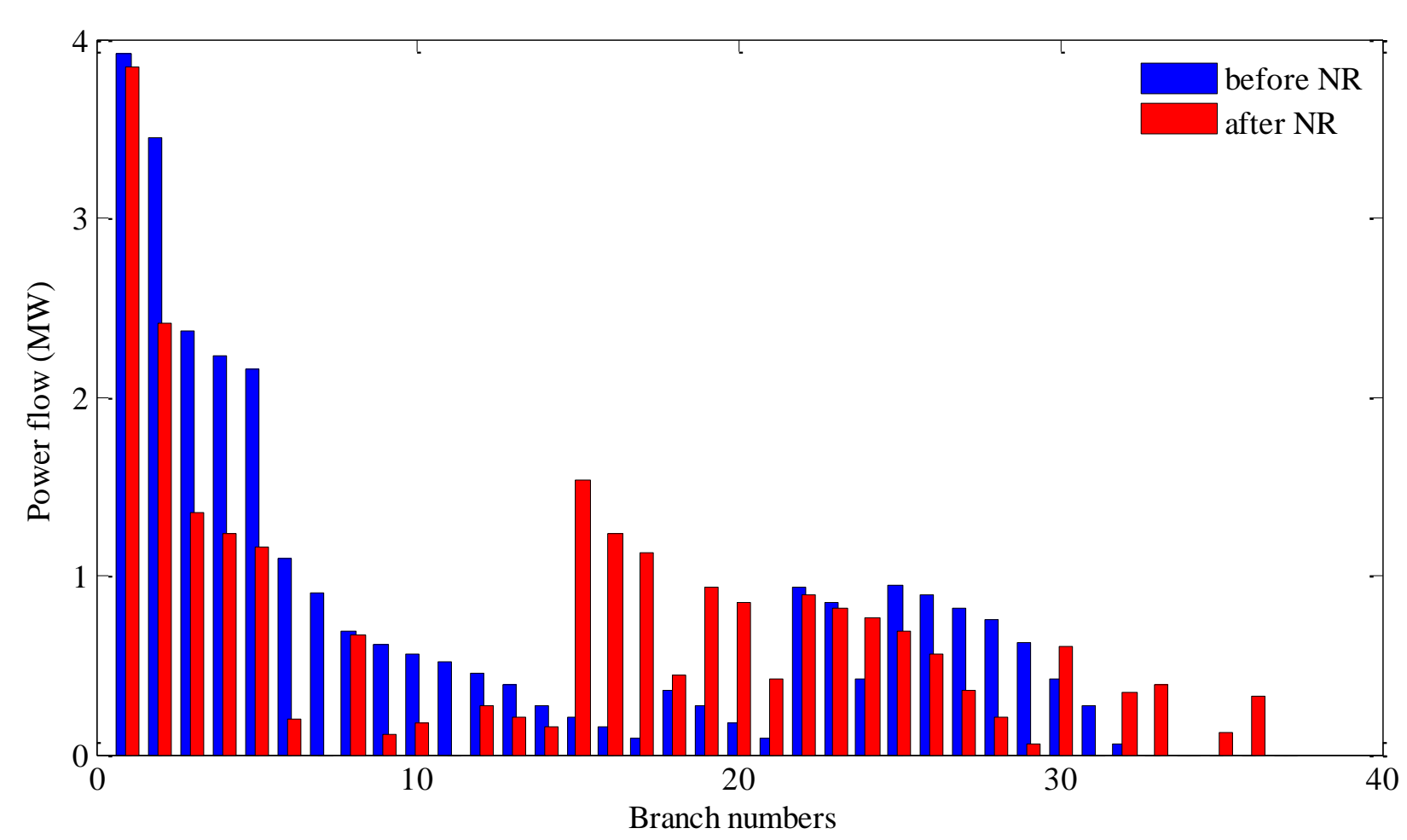

Figure.4 Comparison of branch power flow 


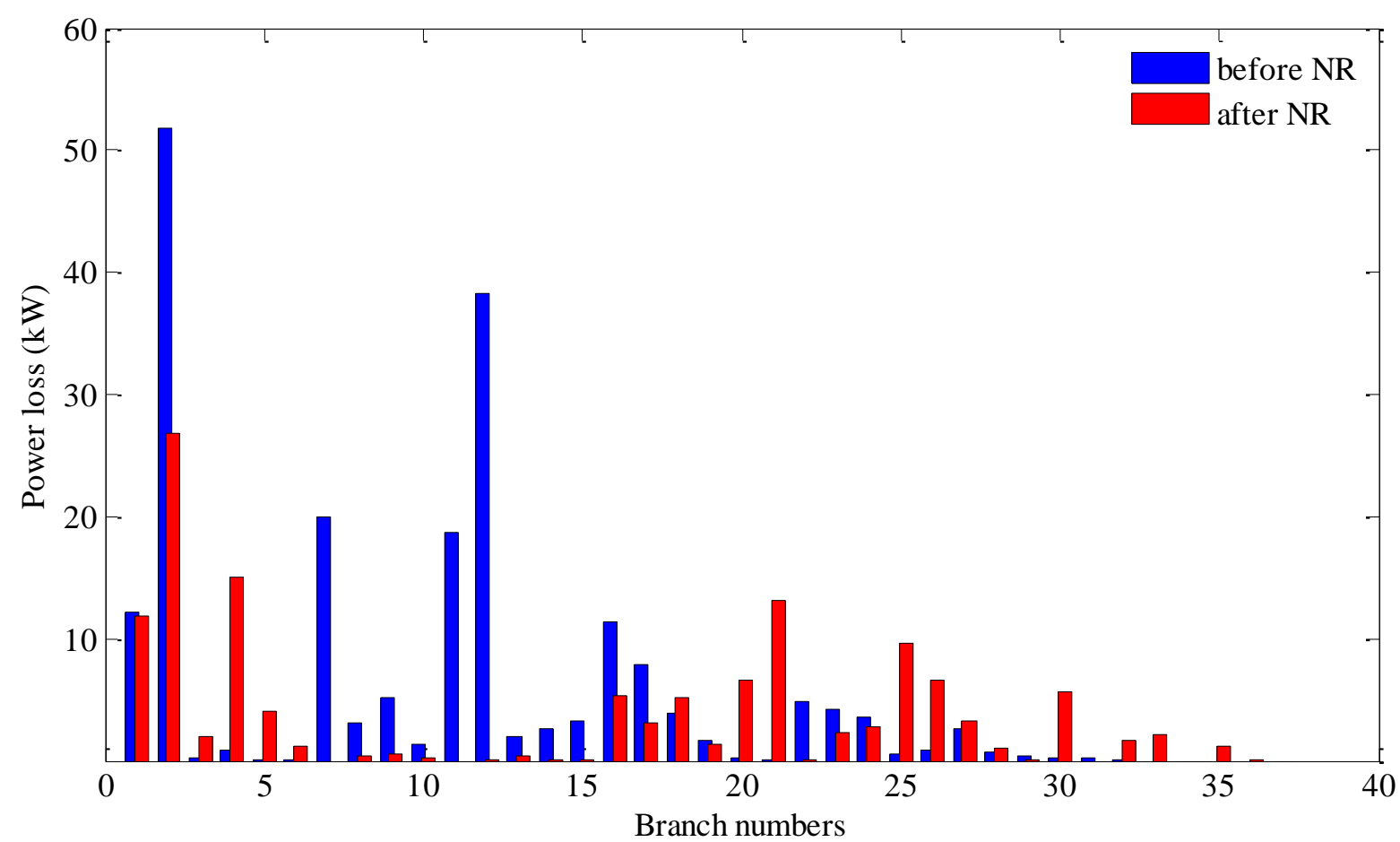

Figure.5 Power loss in various branches

Table 3. Statistical indices of the optimal solutions

\begin{tabular}{|l|c|c|c|c|}
\hline \multirow{2}{*}{ Methods } & \multicolumn{3}{|c|}{ Load balancing index } & \multirow{2}{*}{ Std. Dev. } \\
\cline { 2 - 4 } & Best & Average & Worst & \\
\hline HBB-BC[10] & 0.0534073 & 0.0557439 & 0.0542017 & 0.0011069 \\
\hline AWIDPSO & 0.0338200 & 0.0341300 & 0.0339300 & 0.0002190 \\
\hline
\end{tabular}

\section{Conclusion}

In this paper, a powerful metaheuristic adaptive weighted improved discrete particle swarm optimization algorithm is successfully employed to minimize the load balancing index so as to reduce real power loss and improving the voltage profile. Simulation is carried on 33 bus system and the simulated results are compared with earlier research work.

Therefore, it is concluded that the proposed algorithm robustness and effective alternative for finding global optimum network reconfiguration. It is found that the selection of the tie-switch shows its uniqueness and the obtained configuration is independent of the initial configuration of the network. Further, provides solution to minimize LBI in the radial distribution system and finally, the numerical results would be useful for power distribution companies.

Due to the concern over global warming, penetration of renewable energy resources necessitate. Optimal location and analyze the distribution network in the presence of distributed generation using the proposed algorithm is considered as future scope.

\section{References}

[1] C. Blum and A. Roli, "Metaheuristics in combinatorial optimization overview and conceptual comparison", ACM Computing Surveys, Vol.35, No.3, pp-268-308, 2003.

[2] M. E. Baran and F. F. Wu, "Network reconfiguration in distribution systems for loss reduction and load balancing", IEEE Transactions on Power Delivery, Vol.4, No.2, pp.1401-1407, 1989.

[3] H. D. Chiang and R. J. Jumeau, "Optimal Network Reconfigurations in Distribution Systems: Part 2: Solution Algorithms and Numerical Results", IEEE Transactions on Power Delivery, Vol. 5, No.3, pp. 1568-1574, 1990.

[4] A. Saffar, R. Hooshmand, and A. Khodabakhshian, "A new fuzzy optimal reconfiguration of distribution systems for loss reduction and load balancing using ant colony 
search-based algorithm", Applied Soft Computing, Vol. 11, No.5, pp. 4021-4028, 2011.

[5] P. V. Prasad, S. Sivanagaraju, and N. Sreenivasulu, "Network Reconfiguration for Load Balancing in Radial Distribution Systems Using Genetic Algorithm", Electric Power Components and Systems, Vol. 36, No.1, pp. 6372, 2007.

[6] T. Niknam, "An efficient hybrid evolutionary algorithm based on PSO and HBMO algorithms for multi-objective Distribution Feeder Reconfiguration", Energy Conversion and Management, Vol. 50, No.8, pp. 2074-2082, 2009.

[7] P. V. V. Rama Rao and S. Sivanagaraju, "Radial Distribution Network Reconfiguration for Loss Reduction and Load Balancing using Plant Growth Simulation Algorithm", International Journal on Electrical Engineering and Informatics, Vol. 2, No.4, pp. 266-277, 2010.

[8] T. Niknam, E. Azad farsani, and M. Nayeripour, "An efficient multi-objective modified shuffled frog leaping algorithm for distribution feeder reconfiguration problem", European Transactions on Electrical Power, Vol. 21, No.1, pp. 721-739, 2011.

[9] A. Saffar, R. Hooshmand, and A. Khodabakhshian, "A new fuzzy optimal reconfiguration of distribution systems for loss reduction and load balancing using ant colony search-based algorithm", Applied Soft Computing, Vol. 11, No.5, pp. 4021-4028, 2011.

[10] S. Mostafa, A. Saeedeh, and S. Mohammad, "An Efficient Hybrid Big Bang-Big Crunch Algorithm for Multi-objective Reconfiguration of Balanced and Unbalanced Distribution Systems in Fuzzy Framework", Electric Power Components and Systems, Vol. 41, No.1, pp.7599, 2013.

[11] D. SudhaRani, N. Subrahmanyam, and M. Sydulu, "Multi-Objective Invasive Weed
Optimization -An application to optimal network reconfiguration in radial distribution systems", Electrical Power and Energy Systems, Vol. 73, pp. 932-942, 2015.

[12] S. M. Myint and S. W. Naing, "Network reconfiguration for loss reduction and voltage profile improvement of 110-bus radial distribution system using exhaustive search techniques", International Journal of Electrical and Computer Engineering, Vol. 5, No.4, pp. 788-797, 2015.

[13] S. Mostafa and R. Bakhtiary, "Optimal multiobjective reconfiguration and capacitor placement of distribution systems with the Hybrid Big Bang-Big Crunch algorithm in the fuzzy framework", Ain Shams Engineering Journal, Vol. 7, No.1, pp. 113-129, 2016.

[14] M. N. B. Muhtazaruddin, N. A. Bani, S. A. Mohd Aris, S. Z. A. Jalil, H. M. Kaidi, A. Y. Abd Fatah, J. J. Jamian, F. Muhammad-Sukki, and S. H. Abu-Bakar, "Distribution power loss minimization via distributed generation, capacitor and network reconfiguration", Indonesian Journal of Electrical Engineering and Computer Science, Vol. 5, No.3, pp. 488495, 2017.

[15] K. Rameshkumar, R. K. Suresh, and K. M. Mohanasundaram, " Discrete Particle Swarm Optimization (DPSO) Algorithm for Permutation Flow shop Scheduling to Minimize Make-span", In: Proc. of International Conf. on Natural Computation, Changsha, China, Advances in Natural Computation, LNCS, Vol. 3612, pp. 572-581, 2005.

[16] M. Li, B. Wu, P. Yi, C. Jin, Y. Hu, and T. Shi, "An improved discrete particle swarm optimization algorithm for high-speed trains assembly sequence planning", Assembly Automation, Vol. 33, No. 4, pp. 360 - 373, 2013. 